\title{
Tratamiento endoscópico de adenomas de la papila menor: reporte de dos casos
}

\section{Endoscopic treatment of minor papilla adenomas: Report on two cases}

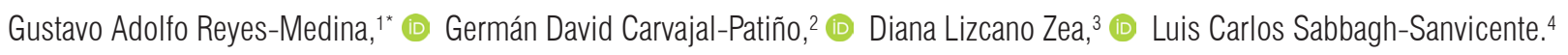

\author{
Gacceso abierto \\ Citación: \\ Reyes-Medina GA, Carvajal-Patiño GD, Lizcano- \\ Zea D, Sabbagh-Sanvicente LC. Tratamiento \\ endoscópico de adenomas de la papila \\ menor: reporte de dos casos. Rev Colomb \\ Gastroenterol. 2021;36(Supl.1):26-29. https://doi. \\ org/10.22516/25007440.514 \\ Médico internista y gastroenterólogo. \\ Departamento de Gastroenterología, Clínica \\ Universitaria Colombia. Fundación Santa Fe de \\ Bogotá. Bogotá, Colombia. \\ 2 Cirujano general, gastroenterólogo. \\ Departamento de Gastroenterología, Clínica \\ Universitaria Colombia. Bogotá, Colombia. \\ 3 Médica patóloga. Departamento de Patología, \\ Clínica Universitaria Colombia. Bogotá, \\ Colombia. \\ ${ }^{4}$ Médico internista y gastroenterólogo. Jefe del \\ servicio de gastroenterología y endoscopia \\ digestiva de Colsanitas. Bogotá, Colombia. \\ *Correspondencia: Gustavo Adolfo Reyes-Medina \\ gustavoareyes@hotmail.com \\ Fecha recibido: $23 / 02 / 20$ \\ Fecha aceptado: $27 / 05 / 20$
}

\section{Resumen}

Existen numerosas publicaciones sobre resección endoscópica de lesiones de la papila mayor, pero solo se han presentado series de casos individuales de resección de lesiones de la papila menor. En el presente artículo se describe el éxito técnico y la seguridad de la resección endoscópica de dos lesiones adenomatosas de la papila menor.

\section{Palabras clave}

Adenoma de la papila menor, papilectomía endoscópica de la papila menor.

\section{Abstract}

There are various publications on endoscopic resection of major papilla lesions, but only individual case series of resection of minor papilla lesions have been reported. This article describes the technical success and safety of endoscopic resection of two adenomatous lesions of the minor papilla.

\section{Keywords}

Minor papilla adenoma; Endoscopic papillectomy of minor papilla.

\section{INTRODUCCIÓN}

Los adenomas de la papila mayor pueden ocurrir esporádicamente o en el contexto de síndromes genéticos como la poliposis adenomatosa familiar y son consideradas lesiones premalignas. La técnica de resección endoscópica de estas lesiones ha sido ampliamente descrita $(1,2)$. En Colombia se han publicado reportes de casos y artículos que describen la experiencia local con respecto a la resección endoscópica de adenomas de la papila mayor $(3,4)$. Los adenomas de la papila menor son lesiones mucho menos frecuentes que las de la papila mayor y solo se han descrito 
en reportes de casos (5-8). Hasta el momento no se han descrito casos de papilectomía endoscópica de la papila menor en Colombia. En este artículo se describen dos casos de pacientes con adenomas de la papila menor tratados por medio de papilectomía endoscópica en la Clínica Universitaria Colombia, en Bogotá.

\section{DESCRIPCIÓN DE CASOS}

\section{Caso 1}

Mujer de 66 años a quien se le realizó esofagogastroduodenoscopia por síntomas dispépticos y se encontró una lesión de aspecto polipoide sésil (Is) de 10 milímetros en la segunda porción duodenal; en la valoración con duodenoscopio de visión lateral se observó que la lesión se encuentra en la papila menor (Figura 1). Las biopsias informaron un adenoma tubular con displasia de bajo grado. La ultrasonografía endoscópica mostró una lesión que compromete hasta la muscularis mucosa, susceptible de resección endoscópica. La colonoscopia total resultó normal, al igual que la colangiorresonancia, no se halló páncreas divisum.

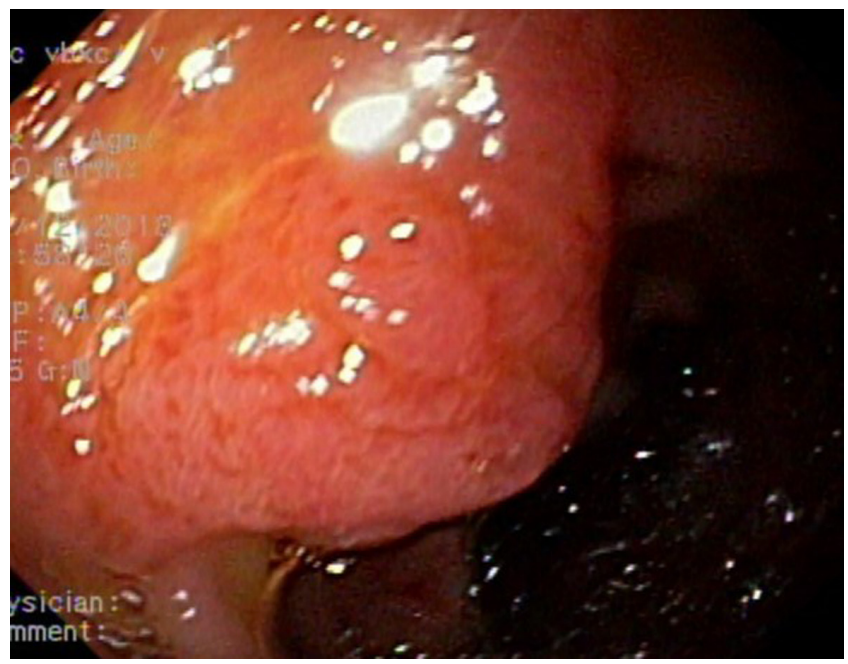

Figura 1. Imagen endoscópica del adenoma de la papila menor.

La paciente fue llevada a ampulectomía de la papila menor. En cuanto a la técnica utilizada, la paciente estuvo bajo anestesia general, en decúbito prono modificado. Con el duodenoscopio se ingresó a la segunda porción duodenal y se examinó la papila mayor, la cual estaba normal. En el eje semilargo se logró enfrentar la papila menor, la cual presentó cambios de aspecto adenomatoso, de 10 milímetros de diámetro mayor. Se elevó la lesión con solución salina normal + azul de metileno (dilución 1:100 000) y se realizó una ampulectomía con asa diatérmica. Se recuperó la muestra con malla y se envió a patología. No hubo compli- caciones inmediatas. En el reporte de patología se registró un adenoma tubular de tipo intestinal con displasia de bajo grado completamente resecado (Figuras 2 y 3 ).

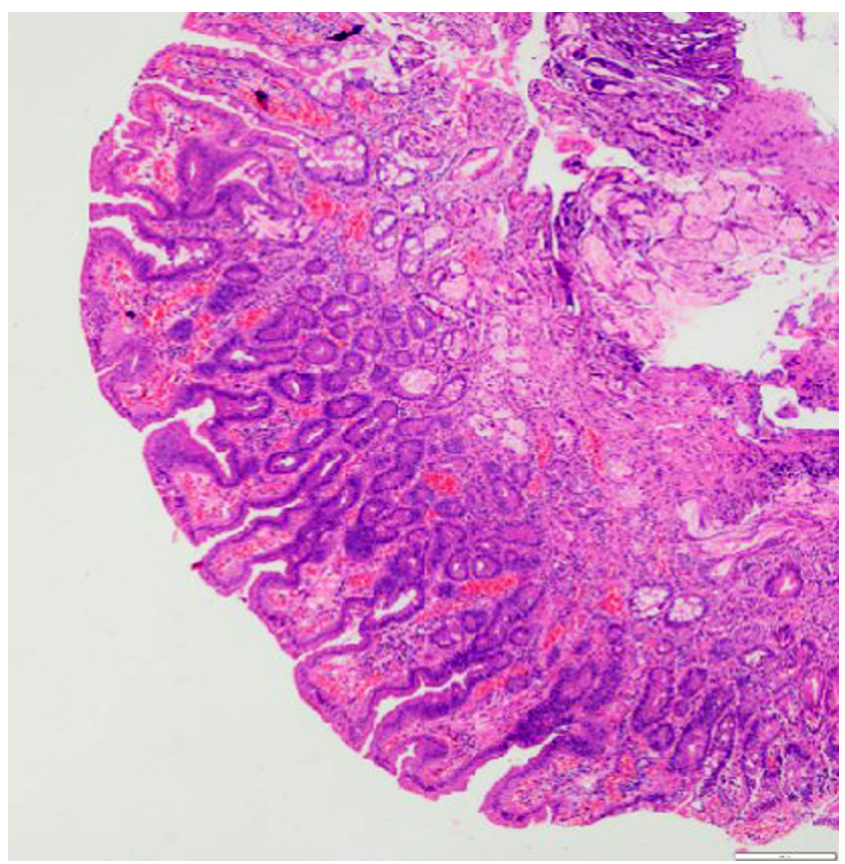

Figura 2. Imagen de histopatología del adenoma resecado, 10x.

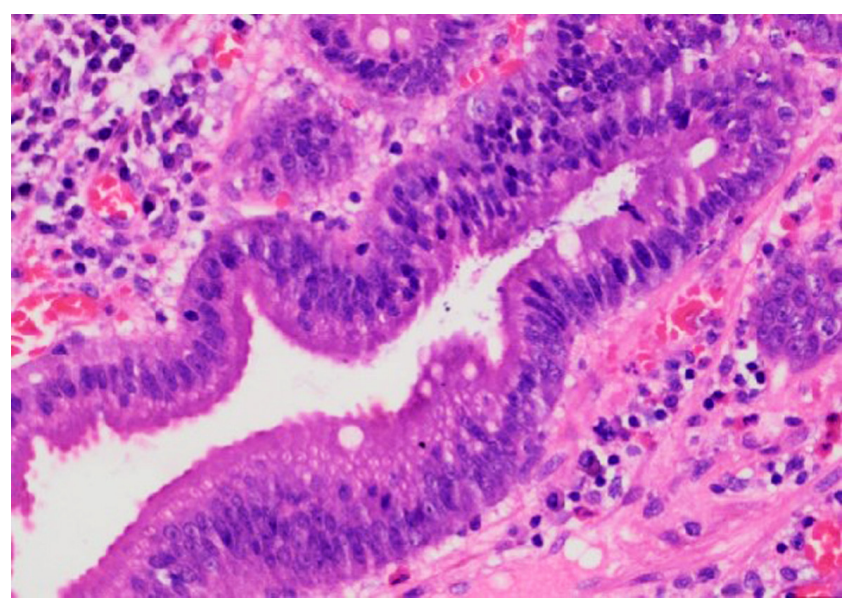

Figura 3. Imagen de histopatología del adenoma resecado, 40x.

La paciente fue hospitalizada el día del procedimiento para observación, la evolución fue satisfactoria y fue dada de alta al día siguiente. En el seguimiento con duodenoscopia realizada a los 6 meses, 12 meses y 24 meses no hubo recurrencia de la lesión.

\section{Caso 2}

Hombre de 73 años con antecedente de disección endoscópica submucosa de lesión de recto (adenoma tubulo- 
velloso con displasia de alto grado), a quien se le realizó esofagogastroduodenoscopia por síntomas dispépticos y se encontró una lesión de aproximadamente 10 milímetros de diámetro mayor de aspecto adenomatoso; en la valoración con duodenoscopio la lesión fue compatible con un adenoma de la papila menor, de aproximadamente 10 milímetros de diámetro (Figura 4), mientras que la papila mayor estaba normal. Las biopsias informaron de un adenoma tubular con displasia de bajo grado. La colangiorresonancia fue normal, sin evidencia de páncreas divisum. El paciente fue llevado a papilectomía de la papila menor, cuya técnica fue similar a la descrita en el caso 1 . No se presentaron complicaciones. En la patología se identificó un adenoma tubular de tipo intestinal con displasia de bajo grado, completamente resecado (Figuras 5 y 6 ).

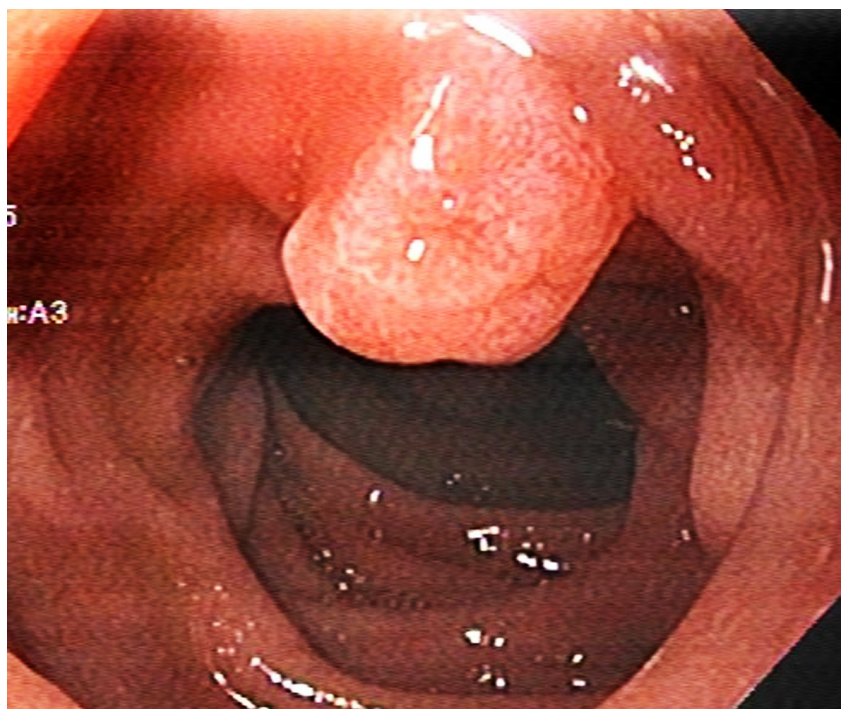

Figura 4. Imagen endoscópica del adenoma de la papila menor.

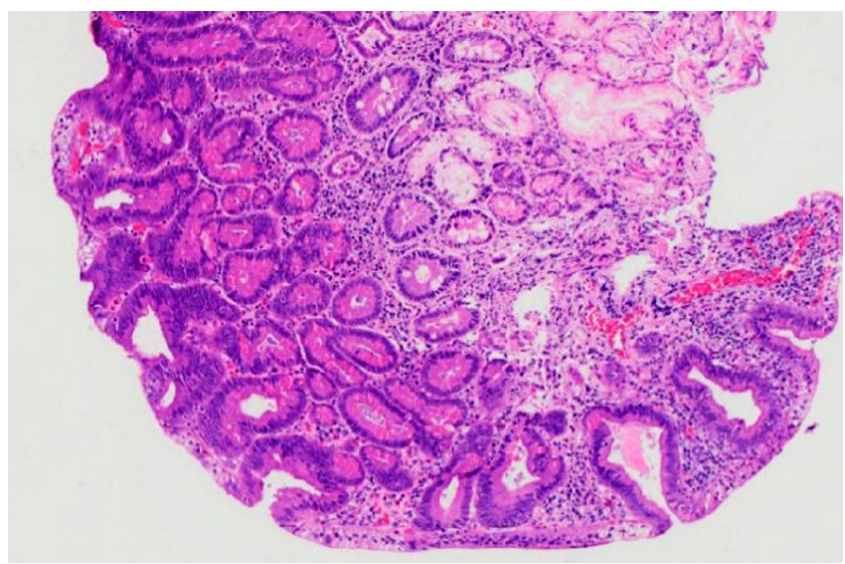

Figura 5. Imagen de histopatología del adenoma resecado, 10x.

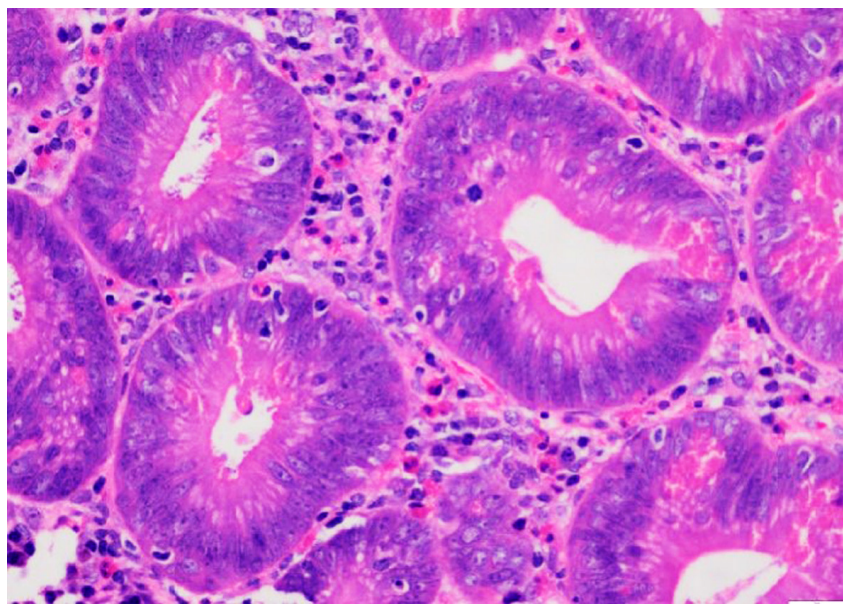

Figura 6. Imagen de histopatología del adenoma resecado, 40x.

El paciente fue hospitalizado el día del procedimiento para observación, la evolución fue adecuada y fue dado de alta al siguiente día. En el seguimiento con duodenoscopia realizada a los 4 meses, 10 meses y 20 meses no hubo recurrencia de la lesión.

\section{DISCUSIÓN}

Las enfermedades de la papila menor se describen infrecuentemente y las lesiones varían entre adenomas benignos (5-8), paragangliomas gangliocíticos $(9,10)$ y carcinomas $(11,12)$. Pueden ser lesiones únicas o asociadas con adenomas de la papila mayor (8) y poliposis adenomatosa familiar (8).

Es necesario definir la anatomía del conducto pancreático antes de la papilectomía por medio de ultrasonografía endoscópica o de colangiorresonancia, y descartar el compromiso del conducto pancreático por el adenoma. Cuando coexiste páncreas divisum, debe insertarse un stent pancreático en el conducto de Santorini después de la papilectomía para disminuir la posibilidad de pancreatitis pos-CPRE (5-8). En ninguno de los dos casos descritos se encontró páncreas divisum, por lo cual no se insertaron stents pancreáticos en el conducto de Santorini y en ninguno de ellos se presentó pancreatitis posresección. En los casos en que se inserta un stent pancreático, se recomienda retirarlo luego de 2 semanas para disminuir el riesgo de lesiones en el conducto pancreático (2).

La infiltración submucosa para levantar la lesión previa a su resección es materia de controversia. Actualmente, para las resecciones de la papila mayor, la mayoría de los autores no utiliza esta medida $(1,2)$. En algunas de las series de papilectomía de la papila menor los autores no la usaron (5), mientras que otros autores sí lo hicieron (6). En nues- 
tros dos casos se realizó infiltración de la submucosa con azul de metileno diluido en solución salina normal, con el fin de delimitar claramente la lesión y disminuir el riesgo de perforación, según la consideración del médico que realizó los procedimientos.

La resección completa del adenoma, en una sola pieza, es la meta de la papilectomía y se logró en nuestros dos casos. La recurrencia posresección se ha reportado entre el $0 \%$ y el $33 \%$ (13) de los adenomas de papila mayor. Al momento de escribir este artículo, no se encontraron datos específicos de recurrencia pospapilectomía de adenomas de la papila menor.

Las recomendaciones con respecto al seguimiento endoscópico para descartar la recurrencia de la lesión varían. Se debe realizar con un duodenoscopio de visión lateral. Kandler y Neuhaus recomendaron hacer el seguimiento cada 3 meses el primer año, luego cada 6 meses por otro año y, finalmente, cada año por 3 años (2). En las guías de la American Society for Gastrointestinal Endoscopy (ASGE) se recomienda hacer seguimiento entre 1 y 6 meses posresección, y luego cada 3 a 12 meses por al menos 2 años (1). Estas recomendaciones son principalmente para el seguimiento pospapilectomía de adenomas de la papila mayor. No hay recomendaciones específicas para seguimiento posresección de adenomas de papila menor.

\section{CONCLUSIONES}

Se presentan dos casos de pacientes a quienes se les realizó papilectomía endoscópica de la papila menor, ambos correspondieron a adenomas tubulares con displasia de bajo grado completamente resecados, no hubo complicaciones relacionadas con el procedimiento y el seguimiento hasta 20 y 24 meses fue negativo para recurrencia.

\section{REFERENCIAS}

1. ASGE Standards of Practice Committee, Chathadi KV, Khashab MA, Acosta RD, Chandrasekhara V, Eloubeidi MA, Faulx AL, Fonkalsrud L, Lightdale JR, Salztman JR, Shaukat A, Wang A, Cash BD, DeWitt JM. The role of endoscopy in ampullary and duodenal adenomas. Gastrointest Endosc. 2015;82(5):773-81. https://doi.org/10.1016/j.gie.2015.06.027

2. Kandler J, Neuhaus H. How to Approach a Patient With Ampullary Lesion. Gastroenterology. 2018;155(6):16701676. https://doi.org/10.1053/j.gastro.2018.11.010

3. Castaño R, Ruiz MH, Sanín E, Erebrie F, Garcia LH, Nuñez E. Experiencia local en la resección endoscópica de la papila. Rev Col Gastroenterol, 2007;22(3):173-89.

4. Solano J, Cabrera LF, Pinto R, López R. Manejo actual del adenoma de la ampolla de Váter. Presentación de caso. Rev Col Cir. 2016;31:212-8.

5. Trevino JM, Wilcox CM, Varadarajulu S. Endoscopic resection of minor papilla adenomas (with video). Gastrointest Endosc. 2008;68(2):383-6. https://doi.org/10.1016/j.gie.2008.03.1070

6. Kanamori A, Kumada T, Kiriyama S, Sone Y, Tanikawa M, Hisanaga Y, Toyoda H, Kawashima H, Itoh A, Hirooka Y, Goto H. Endoscopic papillectomy of minor papillar adenoma associated with pancreas divisum. World J Gastroenterol. 2009;15(9):1138-40. https://doi.org/10.3748/wjg.15.1138

7. Lapp RT, Hutchins GF. Minor Papilla Adenoma Management in Patients with Pancreas Divisum and Familial Adenomatous Polyposis. ACG Case Rep J.
2013;1(1):47-50. https://doi.org/10.14309/cri.2013.17

8. Ahmed M, Philipose J, Hunton A, Andrawes S. Endoscopic Papillectomy for Major and Minor Papillary Adenoma in Familial Adenomatous Polyposis. ACG Case Rep J. 2019;6(3):1-4. https://doi.org/10.14309/crj.0000000000000019

9. Loew BJ, Lukens FJ, Navarro F, Roy M, Mattia A, Howell DA. Successful endoscopic resection of a gangliocytic paraganglioma of the minor papilla in a patient with pancreas divisum and pancreatitis (with video). Gastrointest Endosc. 2007;65(3):547-50. https://doi.org/10.1016/j.gie.2006.07.019

10. Matsubayashi $H$, Ishiwatari $H$, Matsui $T$, Fujie S, Uesaka K, Sugiura T, Okamura Y, Yamamoto Y, Ashida R, Ito T, Sasaki $\mathrm{K}$, Ono H. Gangliocytic Paraganglioma of the Minor Papilla of the Duodenum. Intern Med. 2017;56(9):1029-1035. https://doi.org/10.2169/internalmedicine.56.7812

11. Matsui T, Matsubayashi H, Hotta K, Sasaki K, Ito H, Ono $\mathrm{H}$. A case of carcinoma in an adenoma of the duodenal minor papilla successfully treated with endoscopic mucosal resection. Endosc Int Open. 2016;4(3):E252-4. https://doi.org/10.1055/s-0041-111500

12. Kawashima Y, Ogawa M, Yamaji Y, Kodama T, Yokota M, Kawanishi A, Hirabayashi K, Mine T. A Case of Endoscopic Mucosal Resection of Carcinoma in Adenoma at the Minor Duodenal Papilla. Case Rep Oncol. 2019;12(2):354-363. https://doi.org/10.1159/000499968

13. Pandolfi M, Martino M, Gabbrielli A. Endoscopic treatment of ampullary adenomas. JOP. 2008;9(1):1-8. 